\title{
Identification of O-antigen polymerase transcription and translation start signals and visualization of the protein in Salmonella enterica serovar Typhimurium
}

\author{
Danny K.-H. Wong, Christina Morris, Tin L. Lam, Wan K. R. Wong \\ and Jim Hackett
}

Author for correspondence: Jim Hackett. Tel: +852 2358 7292. Fax: +852 23581552. e-mail: bcjames@usthk.ust.hk

Department of Biochemistry, Hong Kong University of Science and Technology, Clear Water Bay, Kowloon, Hong Kong Special Administrative Region, China

\begin{abstract}
The wzylrfc gene, encoding the O-antigen polymerase, of Salmonella enterica serovar Typhimurium has been previously cloned and sequenced. In the present work, the wzy transcriptional startpoint was initially identified by primer extension. Next, wzy promoter strength in Escherichia coli K-12 was measured, and was found to be greater than that of the induced lac promoter. To define the Wzy translational startpoint, DNA including the wzy promoter and the putative first five residues of the Wzy protein was fused to the $\mathrm{N}$ terminus of glutathione-S-transferase, and the fusion protein purified by affinity chromatography. $\mathrm{N}$-terminal amino acid sequencing yielded the Wzy translational startpoint. Next, the Wzy protein was C-terminally tagged with the FLAG peptide, and immunoblotting of an S. typhimurium strain expressing a low-copy wzy-FLAG gene (five copies per cell) localized the intact Wzy protein in the cytoplasmic membrane of $S$. typhimurium cells. The Wzy protein was not well-expressed from a multi-copy wzy-FLAG ${ }^{+}$plasmid in $S$. typhimurium, or in E. coli K-12.
\end{abstract}

Keywords: Wzy, Rfc, lipopolysaccharide, semi-rough

\section{INTRODUCTION}

The $w z y$ gene (Reeves et al., 1996), which encodes the $47472 \mathrm{Da}$ Wzy protein, was previously cloned and sequenced, from Salmonella enterica serovar Typhimurium (henceforth termed S. typhimurium) (Collins \& Hackett, 1991). The Wzy protein is the O-antigen polymerase. Thus, wzy mutants of $S$. typhimurium have a semi-rough phenotype, in which individual lipid A molecules carry at most a single $\mathrm{O}$-antigen unit. If Wzy is synthesized, the typical O-antigen ladder of wild-type smooth S. typhimurium is seen (Collins \& Hackett, 1991). The $w z y$ gene of S. typhimurium maps in the $35 \cdot 6$ centisome region on the chromosome (Naide et al., 1965), at a site remote from the $r f b$ gene cluster.

The $w z y$ genes of several other enterobacteria have since been cloned and sequenced. Genes analysed in this manner have been identified as $w z y$ genes either on the basis of homology with other $w z y$ genes, or because the

Abbreviation: GST, glutathione-S-tranferase. genes complement mutations that result in semi-rough phenotypes. The wzy genes of various S. enterica serovars have received most attention. It is likely that a close homologue of the S. typhimurium wzy gene is active in S. enterica strains of groups A and D1, as the $S$. typhimurium wzy gene detected homologues in strains of these groups when used as a probe in Southern blotting (Collins \& Hackett, 1991), and the same $\alpha(1,2)$ linkage is present between $\mathrm{O}$-antigen units of strains of groups B (typhimurium), A and D1 (Mäkelä, 1965; Nurminen et al., 1971). A group C2 strain of S. enterica also carries a gene with detectable homology to $S$. typhimurium wzy within a block of group C2-unique genes in the main $r f b$ gene cluster (Brown et al., 1992). A different $w z y$ gene (termed $w z y^{\mathrm{D} 2 \mathrm{E} 1}$ )(Xiang et al., 1994), located in the main $r f b$ gene cluster, has been identified in S. enterica strains of groups D2 and E1 [which have the same $\alpha(1,6)$ O-antigen linkage], partly on the basis of the fact that this is the only gene common to the $r f b$ clusters of strains of these groups to which no $r f b$ function has been assigned (Xiang et al., 1994). However, a wzy gene of S. enterica group D3 $\left(w z y^{\mathrm{D} 3}\right)$ which 
also gives $\alpha(1,6)$ linkage has no detectable sequence similarity to $w z y^{\mathrm{D} 2 \mathrm{E} 1}$ (Curd et al., 1998).

The $w z y$ genes of some smooth strains of Escherichia coli have been identified either by detectable homology to S. typhimurium wzy (Yao \& Valvano, 1994) or by the complementation of mutations in $r f b$ regions (Batchelor et al., 1991; Liu \& Reeves, 1994; Lukomski et al., 1996). Complementation analysis has also indicated that the $w z y$ genes of Shigella dysenteriae (Klena \& Schnaitman, 1993), Shigella flexneri (Morona et al., 1994), Yersinia enterocolitica (Zhang et al., 1996) and Pseudomonas aeruginosa (Coyne \& Goldberg, 1995; De Kievit et al., $1995)$ are located in their respective $r f b$ gene clusters.

Putative Wzy proteins share certain structural features. First, the putative proteins have multiple putative membrane-spanning domains (Collins \& Hackett, 1991; De Kievit et al., 1995; Morona et al., 1994; Xiang et al., 1994; Zhang et al., 1996). The GC content of wzy genes is generally lower than that of the bulk DNA of the bacterium in which the genes are located (Collins \& Hackett, 1991; De Kievit et al., 1995). Analysis of codon usage in $w z y$ genes indicates that 'rare' codons (Grosjean \& Fiers, 1982) are overrepresented, compared to codon usage by other genes sequenced from bacteria containing wzy genes (Collins \& Hackett, 1991; De Kievit et al., 1995; Morona et al., 1994).

Previously (Collins \& Hackett, 1991), it was not possible to view labelled Wzy protein in minicells of an E. coli K12 strain containing a high-copy-number $w z y^{+}$plasmid, nor was Wzy detectable when the $w z y$ gene was under the control of the T7 promoter in a high copy-number plasmid, in an E. coli strain expressing the bacteriophage T7 RNA polymerase. Likewise, expression of the $S$. flexneri Wzy protein could not be detected in E. coli K12 under conditions where the products of adjacent cloned genes were visible after protein labelling (Morona et al., 1994). It has been suggested (Collins \& Hackett, 1991) that the high usage of rare codons in the $w z y$ gene of S. typhimurium may greatly limit Wzy production in E. coli, and the concentration of rare codons in the N-terminal region of the $S$. flexneri $r f c$ gene has been proposed to exert an attenuating effect on Wzy translation (Morona et al., 1994). Indeed, when 2-3 rare codons in the N-terminal region of the $S$. flexneri $w z y$ gene were mutated to synonymous major codons, and the mutated gene then placed under the control of the T7 promoter and the Shine-Dalgarno sequence from gene 10 of phage T7, a low level of Wzy was detected in whole E. coli K-12 cells following induction of the T7 polymerase (provided on a separate plasmid) (Daniels et al., 1998).

Unlike other $w z y$ genes, that of S. typhimurium is not located in the $r f b$ gene cluster, but at a distant site. The S. flexneri wzy gene does not have its own promoter, being part of the $r f b$ operon. Earlier, no attempt was made to determine (other than by sequence inspection) the transcriptional start point of the S. typhimurium $w z y$ gene, and the possibility that wzy might be transcribed by readthrough from a plasmid promoter was not formally excluded. Also, because of the difficulties experienced in obtaining Wzy expression, it was not possible to determine (by $\mathrm{N}$-terminal analysis of purified protein) the translational start point, or to raise antibody against purified Wzy for use in defining the subcellular localization of the protein. Pulse-chase experiments have shown that polymerization of $\mathrm{O}$ specific lipopolysaccharide chains takes place at the periplasmic face of the inner membrane (McGrath \& Osborn, 1991), and it is thus likely that Wzy might be located in the cytoplasmic-membrane fraction. Also, Daniels et al. (1998) constructed (S. flexneri) Wzy-PhoA fusion proteins, and found that fusion proteins with up to $167 \mathrm{aa}$ of Wzy were located in the 'membrane fraction'. The fusion points of expressive lac $Z$ and phoA fusions with $S$. flexneri wzy further indicated that the Wzy protein was located in the cytoplasmic membrane, with 12 transmembrane segments (Daniels et al., 1998). It remains true, however, that a full-length Wzy protein has never been visualized, in either whole cells or the cytoplasmic-membrane fraction. The topics of $S$. typhimurium wzy transcriptional and Wzy translational start signals, the subcellular localization of Wzy and Wzy quantification are addressed in this paper.

\section{METHODS}

Materials. Enzymes active on DNA were obtained from either Gibco-BRL or Boehringer Mannheim and were used as directed by the suppliers. IPTG was purchased from Amersham Pharmacia. $\left[\alpha^{-32} \mathrm{P}\right] \mathrm{dCTP}\left(\sim 1 \cdot 1 \times 10^{14} \mathrm{~Bq} \mathrm{mmol}^{-1}\right)$ and $\left[\gamma_{-}{ }^{32} \mathrm{P}\right] \mathrm{ATP} \quad\left(\sim 1.1 \times 10^{14} \mathrm{~Bq} \mathrm{mmol}^{-1}\right)$ were from Amersham Pharmacia. Oligodeoxynucleotide primers were synthesized by Gibco-BRL. Rabbit anti-FLAG peptide antibodies were the product of Santa Cruz Biotechnology. The secondary antibody for ELISA [phosphatase-labelled goat anti-rabbit IgG (heavy and light chains)] was purchased from Pierce Laboratories. The secondary antibody for immunoblotting (donkey anti-rabbit IgG, conjugated with horseradish peroxidase) was from Amersham Pharmacia. As an ELISA standard, bacterial alkaline phosphatase, C-terminally tagged with the FLAG peptide, was obtained from Eastman Kodak. ELISA plates were purchased from Corning.

Strains and vectors. E. coli K-12 DH5 $\alpha$ (Hanahan, 1983) was the host for recombinant plasmids. Promoter strengths were assessed in the background of E. coli K-12 JM109 (YanischPerron et al., 1985). E. coli BL21(DE3) pLysS (Phillips et al., 1984) was the host for expression of a fusion protein in which part of Wzy was attached to glutathione-S-transferase (GST) (Phillips et al., 1984). S. typhimurium J357 (Cerin \& Hackett, 1989) was used to modify plasmid DNA before transformation into other S. typhimurium strains. S. typhimurium C5 (wildtype) and S. typhimurium LV386 (wzy::IS10) have been described previously (Collins \& Hackett, 1991). The low-copy plasmids pCL1920 (Lerner \& Inouye, 1990), pQF50 (which contains a promoterless lacZ gene after a HindIII insertion site) (Farinha \& Kropinski, 1990), pGEX-2T (Smith \& Johnson, 1988) and pUC18 (Norrander et al., 1983), were used. Plasmid pADE206 $\left(w z y^{+}\right)$has been described previously (Collins \& Hackett, 1991).

Growth media. Minimal M9 medium was prepared as described by Miller (1972) and supplemented prior to use with $\mathrm{MgSO}_{4}\left(0 \cdot 2 \mathrm{mg} \mathrm{ml}^{-1}\right)$, glucose $\left(2 \mathrm{mg} \mathrm{ml}^{-1}\right)$ and thiamin- $\mathrm{HCl}$ 


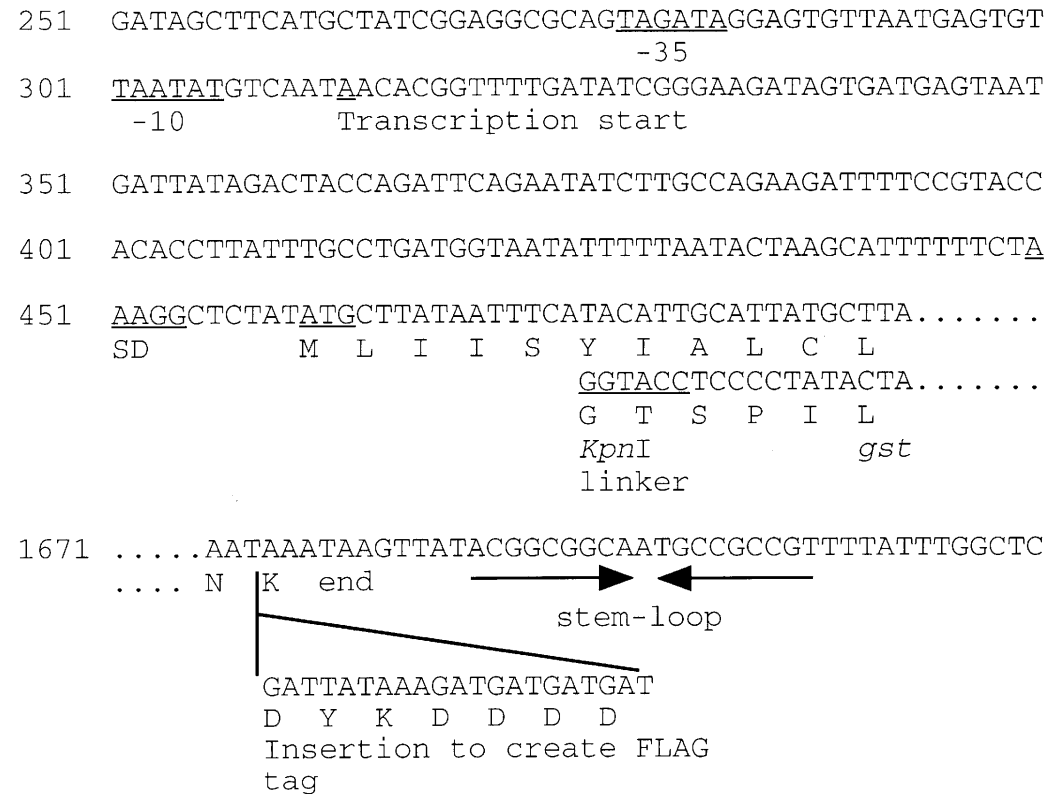

Fig. 1. Constructions in the $\mathrm{N}$ - and $\mathrm{C}$ terminal regions of the wzy gene of $S$. typhimurium, and features of interest in these regions. The nucleotide numbers refer to GenBank submission M60066. The wzy promoter region, the Wzy translational start, the Wzy C-terminus, and the junctions of fusions of Wzy with GST and the FLAG peptide are shown. $\left(50 \mu \mathrm{g} \mathrm{ml} \mathrm{m}^{-1}\right)$. Luria-Bertani (LB) medium was prepared with Bacto tryptone $\left(10 \mathrm{~g} \mathrm{l}^{-1}\right)$, Bacto yeast extract $\left(5 \mathrm{~g} \mathrm{l}^{-1}\right)$ and $\mathrm{NaCl}$ $\left(10 \mathrm{~g} \mathrm{l}^{-1}\right)$. 2XYT medium has been described previously (Collins \& Hackett, 1991). Liquid media were solidified, when required, by the addition of $1.5 \%$ Oxoid bacteriological agar. Bacterial growth was at $37^{\circ} \mathrm{C}$ unless specified otherwise. Ampicillin was added to broth and solid medium at a final concentration of $50 \mu \mathrm{g} \mathrm{ml}^{-1}$. Spectinomycin was used at $50 \mu \mathrm{g}$ $\mathrm{ml}^{-1}$ (E. coli) or $500 \mu \mathrm{g} \mathrm{ml} \mathrm{g}^{-1}$ (S. typhimurium).

Cell fractionation. Cultures $(40 \mathrm{ml})$ were grown in $2 \mathrm{XYT}$ for $3 \mathrm{~h}$. Cells were harvested by centrifugation at $5000 \mathrm{~g}$. The pellet, after washing in $30 \mathrm{mM}$ Tris $/ \mathrm{HCl}, \mathrm{pH} 8 \cdot 1$, was resuspended in $0.8 \mathrm{ml} 20 \%(\mathrm{w} / \mathrm{v})$ sucrose in $30 \mathrm{mM}$ Tris $/ \mathrm{HCl}$, $\mathrm{pH} 8 \cdot 1$. Lysozyme $\left(10 \mathrm{mg} \mathrm{ml}^{-1}\right.$ in $\left.0 \cdot 1 \mathrm{M} \mathrm{EDTA}\right)$ was added to a final concentration of $0.4 \mathrm{mg} \mathrm{ml}^{-1}$. The cells were frozen in an ethanol/dry ice bath for $30 \mathrm{~min}$ and then thawed at room temperature. Twelve millilitres of $3 \mathrm{mM}$ EDTA was added and cells were lysed by sonication (Branson sonicator) until the cell lysate became clear (usually $4 \times 25 \mathrm{~s}$ bursts). Unlysed cells were removed by centrifugation at $5000 \mathrm{~g}$. The lysate was separated into cytoplasmic and total membrane fractions by centrifugation at $200000 \mathrm{~g}$ for $1 \mathrm{~h}$. The resulting pellet (total membrane) was dissolved in PBS $(0.3 \mathrm{ml})$, followed by the addition of sodium lauryl sarcosine (Sarkosyl) in $7 \mathrm{mM}$ EDTA, to $1 \%$. The mixture was incubated at $37^{\circ} \mathrm{C}$ for $30 \mathrm{~min}$ and then centrifuged at $200000 \mathrm{~g}$ for $2 \mathrm{~h}$. The supernatant (Sarkosyl-soluble fraction) is the cytoplasmic-membrane fraction, while the pellet is the outer-membrane fraction. The pellet was washed with $10 \mathrm{mM}$ Tris/ $\mathrm{HCl}, \mathrm{pH} 7.4$ and recentrifuged at $200000 \mathrm{~g}$ for $2 \mathrm{~h}$. The final pellet was then dissolved in PBS.

General analytical methods. DNA manipulations followed established methods (Sambrook et al., 1989). Development of Western blots used the Enhanced Chemiluminescence system (Amersham Pharmacia). SDS-PAGE was usually performed on $5 \%$ (stacking gel) $-12 \%$ (separating gel) polyacrylamide, and proteins were stained with $0 \cdot 25 \%$ Coomassie brilliant blue G250. The gel loading buffer contained 2\% SDS and 100 mM DTT (Sambrook et al., 1989). Lipopolysaccharidespecific silver staining of SDS-PAGE gels was performed as described by Hitchcock \& Brown (1983). The ELISA method has been published by Ausubel et al. (1993). The GST Gene Fusion System was purchased from Amersham Pharmacia. Nterminal protein sequencing was performed at the Biotechnology Laboratory/NAPS Unit, University of British Columbia, Canada.

Accessing the S. typhimurium wzy sequence. When the wzy sequence is mentioned below, the numbers shown are the nucleotide numbers from GenBank submission M60066, where nt 1 is the first nucleotide in the $1750 \mathrm{bp} \mathrm{HindIII}$ fragment carrying the $w z y$ gene of S. typhimurium. Fig. 1 shows the wzy promoter region, the translational start, the Cterminus, and the junctions of fusions of Wzy with GST and the FLAG peptide (see below).

Testing of promoter strength. To assay promoter strengths, PCR fragments, in which various promoters were contained on SphI-HindIII fragments, were cloned into plasmid pQF50. To amplify the $w z y$ promoter, the primers used were prwzyUP1 (5'-251-GATAGCTgCATGCTATCGGAGGCG274-3') and prwzyDOWN1 (5'-344-CATCACaAgCTTCCCGATA-326-3'). The nucleotides in lower case are substitutions in the sequence ( $\mathrm{T}$ to $\mathrm{G}$, nt 258 ; $\mathrm{T}$ to $\mathrm{A}$ and $\mathrm{T}$ to $\mathrm{G}$; nts 336 and 338) to create an SphI site (prwzyUP1) or a HindIII site (prwzyDOWN1). To insert the lac promoter (with the catabolite-activator-protein binding site and lac operator) into pQF50, the primers used were prlacUP (5'-634-GCAGCTGGCAtGcCAGGTTTCCC-612-3') and prlacDOWN (5'-463-TGGTCAaAGCTtTTTCCTGTG-483-3'), where the numbers refer to the sequence of pUC18 (GenBank accession no. L09136) (Norrander et al., 1983). The nucleotides in lower case are substitutions in this sequence ( $\mathrm{C}$ to $\mathrm{T}$, nt 624 and A to C, nt 622; T to A, nt 469 and $G$ to $T$, nt 474) in order to create an SphI site (prlacUP) or a HindIII site (prlacDOWN). To insert the tac promoter into pQF50, the primers used to amplify DNA from plasmid pGEX-2T were prtacUP (5'-65-GGCTGTGCAtGcCGTAAATCAC-86-3') and prtacDOWN (5'-262-GACATGAAgctTGTTTCCTG243-3') where the numbers refer to the GenBank sequence U13850 (Smith \& Johnson, 1988). The bases in lower case are substitutions in the sequence ( $\mathrm{G}$ to $\mathrm{T}$, nt 74 and $\mathrm{T}$ to $\mathrm{C}$, nt 76; TAC to GCT, nts 254-252) in order to create an SphI site (prtacUP) or a HindIII site (prtacDOWN). Transformed 
strains of E. coli K-12 JM109, carrying these plasmids, were grown in M9 minimal medium to $\sim 5 \times 10^{8}$ cells $\mathrm{ml}^{-1}$. Cells were pelleted, resuspended in Z-buffer $(0 \cdot 1-0.5 \mathrm{ml})$ (Miller, 1972) and lysed with 2 drops chloroform and 1 drop $0 \cdot 1 \%$ SDS. The lysates were assayed for $\beta$-galactosidase activity as expressed in Miller units (Miller, 1972).

Fusion of part of wzy with the gst gene. To determine the translation startpoint of the Rfc protein, a portion of the $w z y$ gene, including the $w z y$ promoter and the DNA encoding the first 5 residues of the putative Wzy protein, was fused to the $\mathrm{N}$ terminus of the gst gene. First, an EcoRI-KpnI fragment, containing the required $w z y$ DNA, was cloned into pUC18 and the resulting plasmid then received a KpnI-HindIII fragment containing the required gst DNA, to make plasmid pUST013. The primers used to amplify the $w z y$ DNA were prwzyUP2 (5'-39-TACTTGgAATTCGTCTGTG-57-3') and prwzyDOWN2 (5'-490-GCATAAgGtAccGTATGAAATTAT-467-3'). The nucleotides in lower case are substitutions in this sequence ( $T$ to $G, n t 45 ; T$ to $G$, nt 484 , C to T, nt 482 and AT to CC, nts 480-479) to create an EcoRI site (prwzyUP2) or a KpnI site (prwzyDOWN2). To amplify the gst-containing DNA from plasmid pGEX-2T, primers used were prGSTUP (5'-249-ACAGTAggtAccTCCCCTATACTA-272-3') and prGSTDOWN (5'-1041-ACAGACAAGCTtTGACCGTCTCCG-1018-3'), where the numbers refer to the GenBank sequence U13850 (Smith \& Johnson, 1988). The bases in lower case are substitutions in this sequence (TTC-TG to GGT-CC, nts 255-260 and $G$ to $T$, nt 1030) in order to create a KpnI site (prGSTUP) or a HindIII site (prGSTDOWN). As a result of this construction, the first 5 putative residues of Wzy were fused, via 2 residues encoded by the $K p n I$ site, to the second residue (after M) of GST (Fig. 1).

Primer extension. To determine the transcription startpoint of the $w z y$ gene, the primers used in the primer extension experiment were prwzyDOWN3 (5'-483-GCAATGTATGAAATTATAAGCATATAGAGC-454-3'), prwzyDOWN4 (5'374-TTCTGAATCTGGTAGTCTATAATCATTACTC344-3') and prwzyDOWN5 (5'-405-GGTGTGGTACGGAAAATCTTCTGGCAAGAT-376-3'). These primers were 5' end-labelled with $\left[\gamma^{-32} \mathrm{P}\right]$ ATP using T4 polynucleotide kinase (Gibco BRL) and purified from a polyacrylamide gel. Total RNA was isolated, from strains grown in LB, using a Stratagene Micro RNA Isolation Kit. RNA ( $40 \mu \mathrm{g})$ was mixed with $0.8 \mathrm{pmol}$ purified labelled primer, and the mixture precipitated with $\mathrm{NaCl}$ and ethanol (Sambrook et al., 1989). Reverse transcription was performed using SUPERSCRIPT $\mathrm{RNaseH}^{-}$reverse transcriptase (Gibco BRL), and the annealing and subsequent extension reactions were performed as described in the SUPERSCRIPT $\mathrm{RNaseH}^{-}$reverse transcriptase protocol, except that actinomycin D and RNasin ribonuclease inhibitor (Promega) were added to the reaction

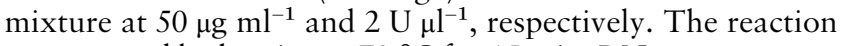
was stopped by heating at $70{ }^{\circ} \mathrm{C}$ for $15 \mathrm{~min}$. RNase treatment, followed by ethanol precipitation, was performed as described by Sambrook et al. (1989). The samples were loaded next to a set of sequencing reactions, with pADE206 as template, which used the same primer. The sequencing reaction was performed using a T7 Sequencing Kit (Amersham Pharmacia).

Insertion of the FLAG tag at the C-terminus of wzy. The FLAG tag (DYKDDDDK) (Hopp et al., 1998) was created at the C-terminus of Wzy by insertion of a heterologous sequence between the penultimate and ultimate residue of Wzy (Fig. 1). PCR primers used were prwzyUP3 (5'-gattacaaagatgatgatgat1679-AAATAAGTTATACGGCGGCAATGCCGCCGTT1709-3') and PrwzyDOWN6 (5'-atcatcatcatctttgtaatc1678-ATTGTTTCTTAGTAAAACGAATCTTATATGC-
1648-3'). The heterologous sequence (gat tac aaa gat gat gat gat) in the primer prwzyUP3 encodes DYKDDDD, while the final $\mathrm{K}$ of the FLAG tag is provided by the ultimate codon (AAA) of the native wzy gene. A sequence complementary to this segment is in the primer prwzyDOWN6. Two PCR reactions were performed, using pADE206 as template. The first reaction used Universal primer (5'-GTAAAACGACGGCCAGT-3', from the Amersham Pharmacia T7 sequencing kit) and prwzyDOWN6, and the other reaction used prwzyUP3 and a primer 5'-TCACACAGGAAACAGCTATGAC-3', which is a modification of the Reverse sequencing primer (Amersham Pharmacia). The two PCR products were mixed and annealed (at the complementing sequences in the primers prwzyUP3 and prwzyDOWN6). The annealed product was used as a template for a final PCR reaction using the Universal and modified Reverse primers. The final product was digested with HindIII and cloned into pUC18 to give pUST011, or into pCL1920 to give pUST012.

\section{RESULTS}

\section{The transcriptional startpoint of the wzy gene}

Cellular RNA from S. typhimurium LV386 (wzy) and the complemented multi-copy $w z y^{+}$strain S. typhimurium LV386/pADE206, was subjected to primer extension from prwzyDOWN3 to localize the wzy transcriptional startpoint (Fig. 2). The wzy transcriptional startpoint was shown to be A313 (Figs 1,2).

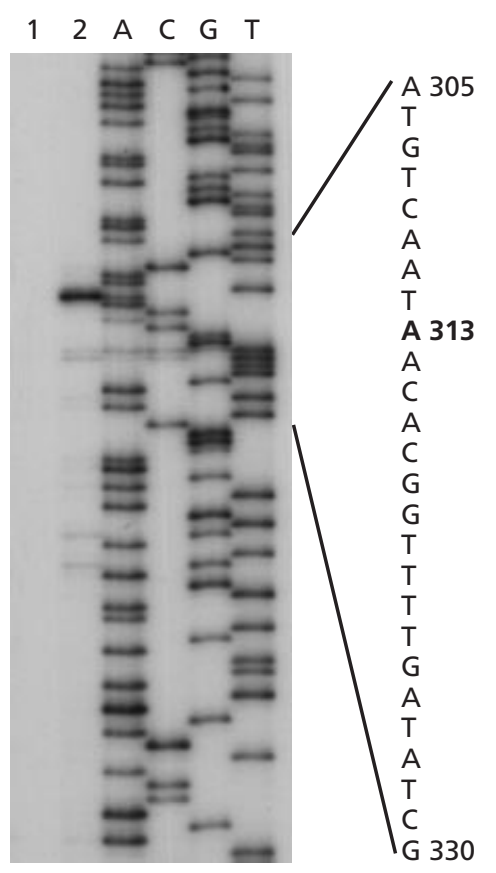

Fig. 2. Identification of the $w z y$ transcription startpoint by primer extension, using the oligonucleotide prwzyDOWN3. RNA $(40 \mu \mathrm{g})$ from $S$. typhimurium LV386 (wzy) (lane 1), and the complemented wzy $^{+}$strain S. typhimurium LV386/pADE206 (lane 2) was annealed with the wzy-complementary primer prwzyDOWN3, and reverse transcription followed (see Methods). A sequencing reaction, using the same primer, was also performed. The letters on the right show DNA sequence, and the bold $A$ at position 313 shows the wzy transcription startpoint. 


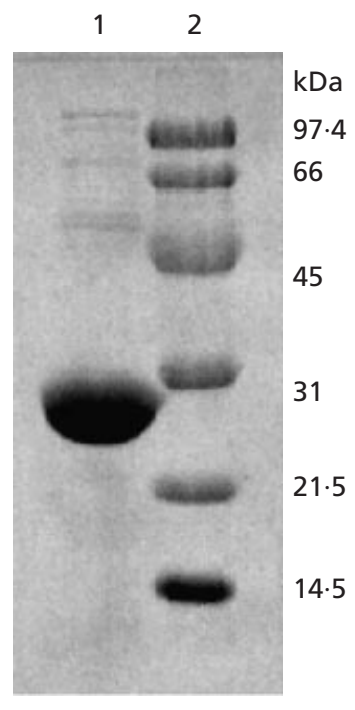

Fig. 3. Purification of the Wzy-GST fusion protein. The wzy promoter and the putative first 5 residues of Wzy were fused to the second residue of GST. The fusion protein was purified by affinity chromatography using agarose-bound glutathione and subsequent glutathione elution (Smith \& Johnson, 1988). Lane 1, purified Wzy-GST $(50 \mu \mathrm{g})$; lane 2, molecular-mass markers (sizes, in $\mathrm{kDa}$, are shown on the right).

Primer extension from two other primers, prwzyDOWN4 and prwzyDOWN5, confirmed this (data not shown). This indicated that the S. typhimurium wzy gene does indeed have its own promoter as previously suggested (Collins \& Hackett, 1991), and allowed the -35 and -10 promoter regions to be positively identified (Fig. 1). Both the -10 signal (TAATAT, nts 301-306) and the -35 signal (TAGATA, nts 278-283) have 4/6 nucleotides of the canonical sequences (TATAAT and TTGACA, respectively) (Hawley \& McClure, 1983; Rosenberg \& Court, 1979).

To measure the strength of the $w z y$ promoter, the $w z y$ promoter region, and control lac and tac promoters, were inserted upstream of the promoterless lacZ gene of plasmid pQF50, and the transcriptional efficiency of the LacZ gene product measured (see Methods). The strength of the $w z y$ promoter was 73.0-99.9 Miller units in two tests: it was thus stronger than the induced lac promoter (30.6-38.0 Miller units) but much weaker than the tac promoter (665-689 Miller units).

\section{The translational startpoint of Wzy}

Initial attempts to purify the Wzy protein, from E coli K12 or S. typhimurium cells with the $w z y$ gene in low- or multi-copy plasmids, were unsuccessful, possibly due to the fact that the protein is membrane associated (see below). A different approach to determination of the $\mathrm{N}$ terminal amino acid sequence of Wzy was therefore taken. Routinely, all or part of foreign proteins may be fused to the C-terminus of GST without affecting the ability of GST to bind glutathione, so that the fusion

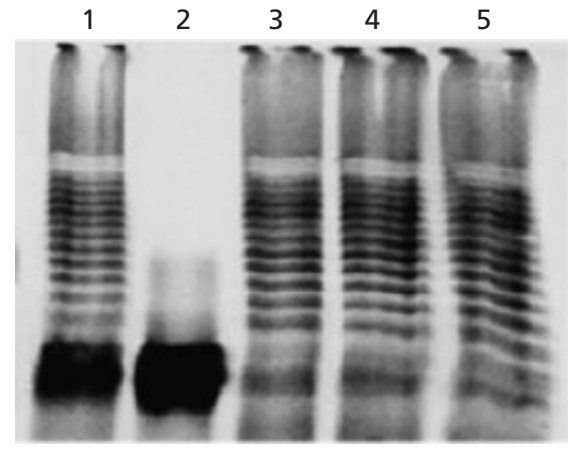

Fig. 4. Lipopolysaccharides of S. typhimurium strains on an SDSPAGE gel, with lipopolysaccharide-specific silver staining. Each lane received material from $10^{7}$ cells grown in $2 X Y T$. Lane $1, S$. typhimurium C5 (wild-type); lane 2, S. typhimurium LV386 (wzy); lane 3, S. typhimurium LV386/pUST010 (low-copy wzy ; the wzy $^{+}$Hindlll fragment was inserted into $\mathrm{pCL1920);} \mathrm{lane} \mathrm{4,} \mathrm{S.}$ typhimurium LV386/pUST011 (multi-copy wzy-FLAG); lane 5, S. typhimurium LV386/pUST012 (low-copy wzy-FLAG).

proteins may be purified by affinity chromatography. It was thought that it might be possible to fuse a small portion of a foreign protein to the $\mathrm{N}$ terminus of GST, also without affecting the glutathione-binding capacity of GST. Accordingly, the entire wzy promoter and a sequence containing the first 5 putative residues of Wzy was fused to the second residue (thus, the starting $\mathrm{M}$ was omitted) of GST (see Methods) (Fig. 1), and a fusion protein sought by affinity chromatography (Fig. 3). This approach was successful. From an $800 \mathrm{ml}$ culture of E. coli BL21(2DE3) pLysS/pUST013, a total of $3.6 \mathrm{mg}$ Wzy-GST fusion protein was obtained. A portion of this protein was subjected to N-terminal amino acid sequencing and the first 10 residues were LIISGTSPIL (Fig. 1). This identifies ATG461-463 as the Wzy start codon (Fig. 1), as previously suggested (Collins \& Hackett, 1991). The sequence AAAGG450-454 (Fig. 1) may, as indicated previously (Collins \& Hackett, 1991), serve as part of a ribosome-binding site upstream of the Wzy start codon.

\section{Visualization of the Wzy protein in cells of S. typhimurium}

Initially, it was planned to purify the Wzy protein so that anti-Wzy antibody might be raised. Fusion proteins with all or part of Wzy linked to the C-terminus of GST were uniformly unstable, and were obtained in only small and variable yields. Accordingly, the FLAG tag was inserted at the C-terminus of Wzy (see Methods) (Fig. 1). This insertion did not obviously affect Wzy function (Fig. 4), as the typical O-antigen ladder was obtained when the wzy strain, S. typhimurium LV386, was complemented with the $w z y-F L A G$ gene, resident in either multi- or low-copy plasmids.

Next, FLAG-tagged Wzy protein, expressed in the $S$. typhimurium LV386-based strains containing either the multi- or low-copy $w z y-F L A G$ plasmids, was sought by 

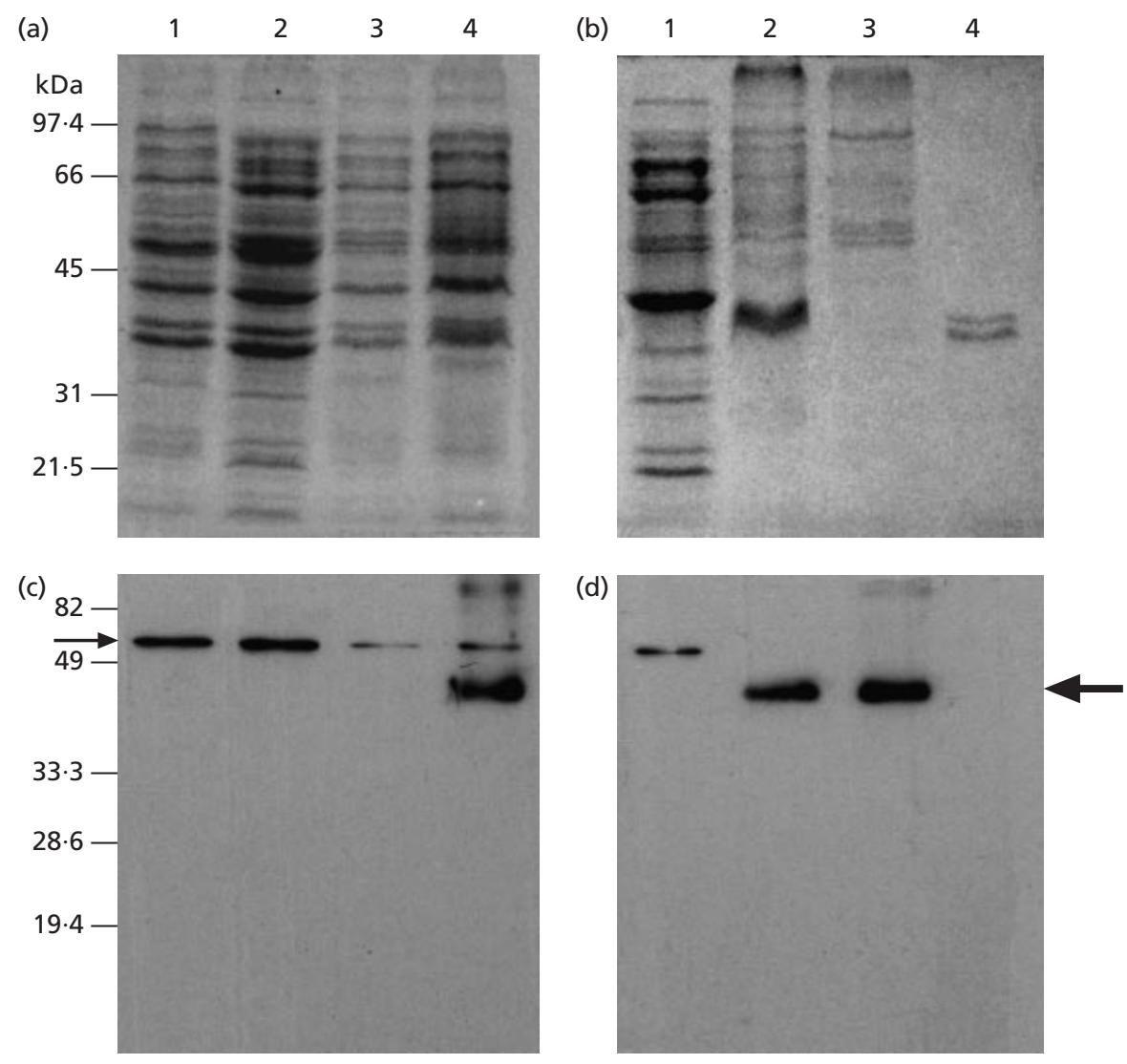

\begin{abstract}
Fig. 5. Detection of the Wzy protein by immunoblotting. For panels (a) and (c) ( $10^{8}$ cells per track), whole cells, grown in LB, were solubilized and analysed by SDS-PAGE. Panel (a) was developed with Coomassie blue and panel (c) by immunoblotting, using antibodies against the FLAG tag $\left(1: 500\right.$ dilution; $0.2 \mu \mathrm{g}$ protein $\left.\mathrm{ml}^{-1}\right)$ as the primary antibody, and the secondary antibody at a 1:1000 dilution. Lanes 1, S. typhimurium C5 $\left(w z y^{+}\right)$; lanes 2, S. typhimurium LV386 (wzy); lanes 3, S. typhimurium LV386/pUST011 (multi-copy wzy-FLAG); lanes 4, S. typhimurium LV386/pUST012 (low-copy wzy-FLAG). For panels (b) and (d), whole cells of S. typhimurium LV386/pUST012 (low-copy wzy-FLAG), grown in LB broth, were fractionated (material from $10^{7}$ cells per track) before SDS-PAGE. Lanes 1, cytoplasmic fraction; lanes 2, whole membrane; lanes 3, cytoplasmic membrane; lanes 4, outer membrane. Panel (b) was developed with Coomassie blue, and panel (d) by immunoblotting, using antibodies against the FLAG tag as the primary antibody. The positions of molecular mass markers, with sizes, are shown on the left. The small arrow (left) marks the position of a cytoplasmic protein, present in all strains, which cross-reacts with the anti-FLAG antibodies. The large arrow (right) shows the FLAGtagged Wzy protein in whole cells, whole membrane, and the cytoplasmic membrane of S. typhimurium LV386/pUST012.
\end{abstract}

immunoblotting (Fig. 5). No genuine FLAG signal was obtained on immunoblots of whole cells of either $S$. typhimurium C5 or S. typhimurium LV386 (neither strain expresses a FLAG peptide), although a protein cross-reactive with the anti-FLAG antibodies was present in all strains examined (and is shown, below, to be located in the cytoplasm of S. typhimurium LV386/pUST012). Surprisingly, a genuine FLAG signal was also absent upon immunoblots of whole cells of $S$. typhimurium LV386/pUST011 (which carries multicopy $w z y-F L A G)$, even though such cells clearly expressed active Wzy protein (Fig. 4). The low level of Wzy expression (below the sensitivity level of the immunoblot) in this strain will be discussed later. When whole cells of S. typhimurium LV386/pUST012 (which has low-copy $w z y-F L A G)$ were examined, however, FLAGtagged Wzy protein was visible (Fig. 5c, track 4), and cell fractionation localized the protein to the cytoplasmic membrane (Fig. 5d, track 3).
In an effort to quantify the Wzy protein in $S$. typhimurium LV386/pUST012, whole cells of this strain, and of control S. typhimurium LV386, were disrupted by boiling in gel-loading buffer, and then used to coat wells of a 96-well tray. An ELISA assay, using a commercial pure FLAG-tagged protein (similarly boiled) as standard, followed (Fig. 6). The Wzy protein level in the (low-copy wzy) strain of $S$. typhimurium LV386/pUST012 was $\sim 25 \mathrm{ng}$ per $10^{7}$ cells (after correction for cross-reaction between the anti-FLAG antibodies and a cytoplasmic protein present in both control and test strains). This calculation assumes that the efficiency of binding to the plastic tray of the FLAGtagged Wzy protein from solubilized whole cells, was the same as that of the pure FLAG-tagged protein standard, and that the FLAG tag does not affect (inhibit?) the natural degradation rate of the unmodified Wzy protein. If these two conditions are met, it may be calculated that when the $w z y$ gene is present at 5 copies 

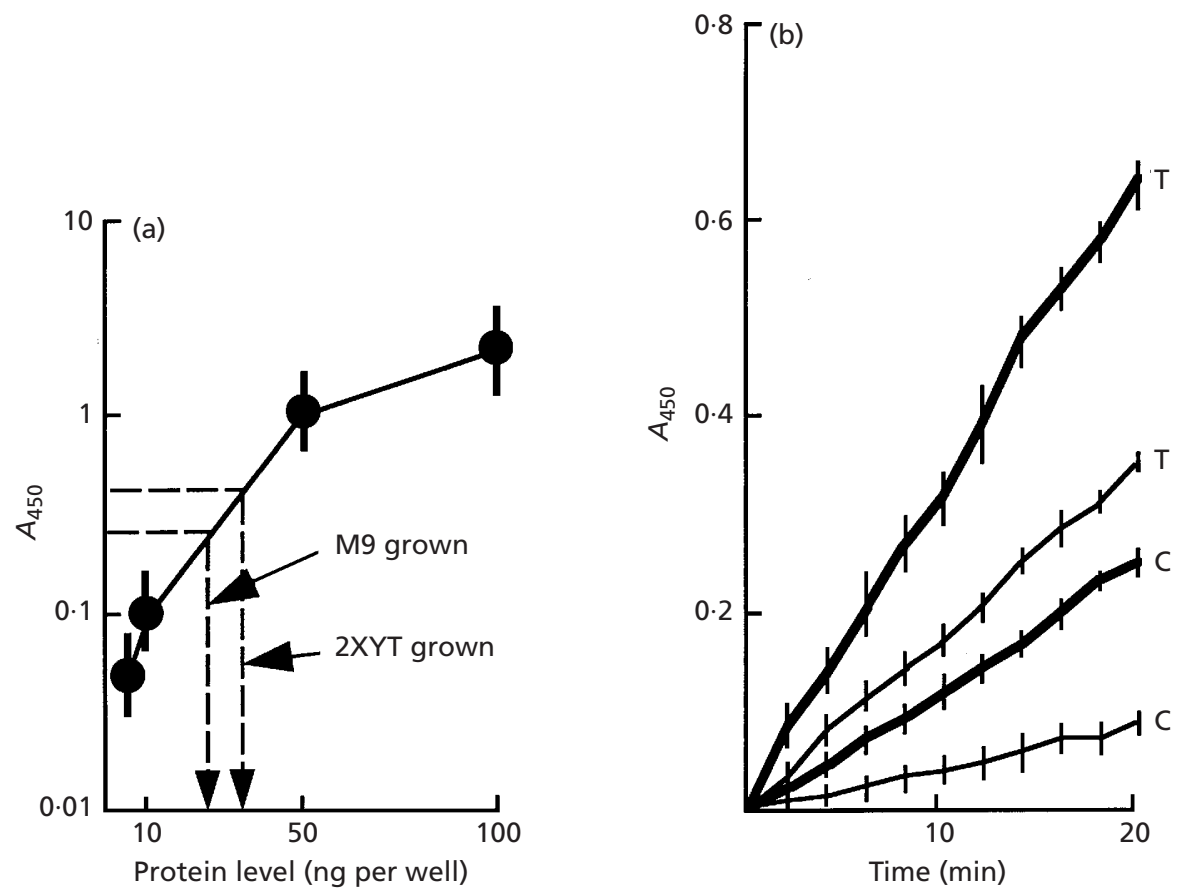

Fig. 6. Quantification of the Wzy protein by ELISA. (a) FLAG-tagged standard protein solubilized in gel-loading buffer was used to coat duplicate wells of a 96-well tray at 5, 10, 50 and 100 ng protein per well, and an ELISA assay (using the commercial anti-FLAG antibodies at a dilution of 1/200) followed. The $A_{450}$ development over 20 min was monitored (and was linear for all standards during this time). The mean absorbancies, with the ranges, are shown. (b) Whole cells of $S$. typhimurium LV386 and S. typhimurium LV386/pUST012, grown in either 2XYT broth (thick lines) or M9 broth (thin lines) were solubilized in gel-loading buffer and the material from $10^{7}$ cells used to coat duplicate wells of a 96-well tray; the $A_{450}$ development over $20 \mathrm{~min}$ is shown (means and ranges). The control (S. typhimurium LV386) $A_{450}$ levels at 20 min were subtracted from those of $S$. typhimurium LV386/pUST012, and the figures obtained then interpolated on the standard curve of (a), to yield estimates of the amount of Wzy-FLAG protein per $10^{7}$ cells. T, test $\left(w z y^{+}\right)$; C, control $\left(w z y^{-}\right)$.

per cell, each cell contains $\sim 30000$ copies of the Wzy protein, which would thus comprise $\sim 1.4 \%$ of total cellular protein (Neidhardt \& Umbarger, 1996). As the Wzy protein is a cytoplasmic-membrane protein, it is relevant to mention that this would equate to $\sim 1$ molecule of Wzy protein for every 700 molecules of membrane phospholipid (Neidhardt \& Umbarger, 1996). If it is assumed that Wzy production from a single $w z y$ gene copy would be one-fifth of the amount seen from the low copy-number $w z y^{+}$plasmid, then Wzy would be present, in wild-type $S$. typhimurium, at $\sim 6000$ copies per cell, comprising $\sim 0.3 \%$ of total protein: one Wzy molecule for each 3500 molecules of phospholipid.

\section{DISCUSSION}

\section{Subcellular localization of Wzy}

Pulse-chase experiments have previously shown that polymerization of O-specific lipopolysaccharide chains takes place at the periplasmic face of the inner membrane (McGrath \& Osborn, 1991), and it was thus always likely that Wzy might be located in the cytoplasmicmembrane fraction. Daniels et al. (1998) located a ( $S$. flexneri) Wzy-PhoA fusion with 167 aa of Wzy in a 'membrane fraction'. It was not possible to view fusions with longer Wzy segments, and it was suggested that this was due to the increasing number of rare codons in the longer wzy segments of such fusions, with consequent poor fusion-protein expression. Also, the locations of expressive lac $Z$ and pho $A$ fusions in the $w z y$ gene were indicative of a cytoplasmic-membrane location for Wzy, with 12 transmembrane segments (Daniels et al., 1998).

The results in this paper are in full agreement with these data. In this work, the full-length S. typhimurium Wzy protein is shown to be located, by immunoblotting of an attached FLAG tag, in the cytoplasmic membrane.

\section{Transcription of wzy}

When an extract of (wild-type) S. typhimurium C5, which contains a single copy of the $w z y$ gene, was used for primer extension, no labelled product was detectable on a gel such as that shown in Fig. 2 (data not shown). The sensitivity of this method thus requires that the $w z y$ gene be present in a multi-copy state. Transcription of the $w z y$ gene from a single startpoint in the multi-copy $w z y^{+}$plasmid, pADE206, was noted in S. typhimurium (Fig. 2), and this transcript was clearly translated into functional Wzy protein (Collins \& Hackett, 1991). It is reasonable to assume that the transcription startpoint of $w z y$ in pADE206 is the same as that used in wild-type 
S. typhimurium. It was further shown that the $w z y$ gene has its own strong promoter.

\section{Level of translation of Wzy}

Previous attempts (Collins \& Hackett, 1991) to detect Wzy translation used systems (maxicells, minicells, T7polymerase-mediated transcription from a T7 promoter) requiring that Wzy be translated in E. coli K-12; as suggested (Collins \& Hackett, 1991), it seemed possible that such expression might be suboptimal, as the Wzy protein has no obvious function in E. coli K-12. This suggestion may have merit. Thus, when pUST012 (which allows good levels of Wzy protein expression in a S. typhimurium background; Fig. 6) was transformed into various E. coli K-12 strains and Wzy protein sought, after SDS-PAGE of lysed whole cells, by immunoblotting with anti-FLAG antibodies, no Wzy protein was detected (data not shown). The absence of obvious Wzy in E. coli K-12 is not due to poor expression of the $w z y$ promoter in E. coli $\mathrm{K}-12$. It may be that the lack of function for Wzy in E. coli K-12 causes the protein to be targeted by the protein-degradation systems of the cell, resulting in a steady-state level of Wzy which is very low or zero.

It was also suggested previously (Collins \& Hackett, 1991) that the presence of many attenuating codons in wzy might cause Wzy to be translated at a low level. With the multi-copy $w z y^{+}$plasmid, pUST011, in $S$. typhimurium, no Wzy protein was seen, and it is possible that the simultaneous initiation of many Wzy protein molecules, with consequent high demand for attenuating tRNA species, results in completion of only a few Wzy protein molecules, so that Wzy protein levels are below the detection level of the immunoblot (Fig. 5) but still adequate to complement the chromosomal wzy mutation (Fig. 4). Daniels et al. (1998) placed the S. flexneri $w z y$ gene downstream of the T7 promoter and the Shine-Dalgarno sequence from gene 10 of phage T7, induced the T7 polymerase, and found that expression of labelled Wzy increased (from undetectable to weak) when 2-3 rare codons in the $\mathrm{N}$-terminal region of $w z y$ were mutated to major synonyms. This is the most direct evidence that translation attenuation by rare codons is a controlling factor in Wzy production.

If only a few Wzy molecules are being synthesized at any time (from the low-copy $w z y^{+}$plasmid pUST012), the protein completion rate may be much higher than seen when $w z y$ is present in a high copy-number plasmid (Fig. 5). This greater Wzy protein completion rate, coupled with possible good stability of the completed protein (embedded, and active, in the cytoplasmic membrane), appears to result in a steady-state level of Wzy equivalent to $\sim 0.3 \%$ of bacterial protein $/ w z y$ copy, in S. typhimurium.

The main conclusions of this study are that the wzy transcriptional startpoint has been identified at residue A313 and confirms that the S. typhimurium wzy gene has its own promoter, with its -10 and -35 sequences, as predicted previously (Collins \& Hackett, 1991). The $w z y$ promoter strength was assessed and lies between that of the lac and tac promoters. N-terminal amino acid sequencing of a Wzy-GST fusion protein confirmed the translation start point which agreed with sequence prediction. Using FLAG as an antibody-reactive tag, a Wzy-FLAG protein was visualized by SDS-PAGE and was localized to the inner membrane. The level of Wzy in wild-type S. typhimurium was estimated to be $\sim 6000$ copies/cell by ELISA analysis.

\section{ACKNOWLEDGEMENTS}

J.H. and C.M. acknowledge the support of the Hong Kong Government Research Grants Council.

\section{REFERENCES}

Ausubel, F. M., Brent, R., Kingston, R. E., Moore, D. D., Seidman, J. G., Smith, J. A. \& Struhl, K. (1993). Current Protocols in Molecular Biology, vol. 2, pp. 11.2.1-11.2.5. New York: John Wiley.

Batchelor, R. A., Haraguchi, G. E., Hull, R. A. \& Hull, S. I. (1991). Regulation by a novel protein of the bimodal distribution of lipopolysaccharide in the outer membrane of Escherichia coli. J Bacteriol 173, 5699-5704.

Brown, P. K., Romana, L. K. \& Reeves, P. R. (1992). Molecular analysis of the $r f b$ gene cluster of Salmonella serovar muenchen (strain M67): the genetic basis of the polymorphism between groups C2 and B. Mol Microbiol 6, 1385-1394.

Cerin, H. \& Hackett, J. (1989). Molecular cloning and analysis of the incompatibility and partition functions of the virulence plasmid of Salmonella typhimurium. Microb Pathog 7, 85-99.

Collins, L. V. \& Hackett. J. (1991). Molecular cloning, characterization, and nucleotide sequence of the $r f c$ gene, which encodes an O-antigen polymerase of Salmonella typhimurium. J Bacteriol 173, 2521-2529.

Coyne, M. J., Jr \& Goldberg, J. B. (1995). Cloning and characterization of the gene $(r f c)$ encoding O-antigen polymerase of Pseudomonas aeruginosa PAO1. Gene 167, 81-86.

Curd, H., Liu, D. \& Reeves, P. R. (1998). Relationships among the $\mathrm{O}$-antigen gene clusters of Salmonella enterica groups B, D1, D2, and D3. J Bacteriol 180, 1002-1007.

Daniels, C., Vindurampulle, C. \& Morona, R. (1998). Overexpression and topology of the Shigella flexneri O-antigen polymerase (Rfc/Wzy). Mol Microbiol 28, 1211-1222.

De Kievit, T. R., Dasgupta, T., Schweizer, H. \& Lam, J. S. (1995). Molecular cloning and characterization of the $r f c$ gene of Pseudomonas aeruginosa (serotype 05). Mol Microbiol 16, 565-574.

Farinha, M. A. \& Kropinski, A. M. (1990). Construction of broadhost-range plasmid vectors for easy visible selection and analysis of promoters. J Bacteriol 172, 3496-3499.

Grosjean, H. \& Fiers, W. (1982). Preferential codon usage in prokaryotic genes: the optimal codon-anti-codon energy and the selective codon usage in efficiently expressed genes. Gene 18, 199-209.

Hanahan, D. (1983). Studies on transformation of Escherichia coli with plasmids. J Mol Biol 166, 557-580.

Hawley, D. K. \& McClure, W. R. (1983). Compilation and analysis of Escherichia coli promoter DNA sequences. Nucleic Acids Res $11,2237-2255$. 
Hitchcock, P. J. \& Brown, T. M. (1983). Morphological heterogeneity among Salmonella lipopolysaccharide chemotypes in silver-stained polyacrylamide gels. J Bacteriol 154, 269-277.

Hopp, T. P., Prickett, K. S., Price, V. L., Libby, R. T., March, C. J., Cerretti, D. P., Urdal, D. L. \& Conlon, P. J. (1998). A short polypeptide marker sequence useful for recombinant protein identification and purification. Biotechnology 6, 1204-1210.

Klena, J. D. \& Schnaitman, C. A. (1993). Function of the $r f b$ gene cluster and the $r f e$ gene in the synthesis of $\mathrm{O}$ antigen by Shigella dysenteriae 1. Mol Microbiol 9, 393-402.

Lerner, C. G. \& Inouye, M. (1990). Low copy number plasmids for regulated low-level expression of cloned genes in Escherichia coli with blue/white insert screening capability. Nucleic Acids Res 18, 463.

Liu, D. \& Reeves, P. R. (1994). Escherichia coli K12 regains its O antigen. Microbiology 140, 49-57.

Lukomski, S., Hull, R. A. \& Hull, S. I. (1996). Identification of the O antigen polymerase $(r f c)$ gene in Escherichia coli $\mathrm{O} 4$ by insertional mutagenesis using a nonpolar chloramphenicol resistance cassette. J Bacteriol 178, 240-247.

McGrath, B. C. \& Osborn, M. J. (1991). Localization of the terminal steps of $\mathrm{O}$-antigen synthesis in Salmonella typhimurium. J Bacteriol 173, 649-654.

Mäkelä, P. H. (1965). Inheritance of the O antigens of Salmonella groups B and D. J Gen Microbiol 41, 57-65.

Miller, J. H. (1972). Experiments in Molecular Genetics. Cold Spring Harbour, NY: Cold Spring Harbour Laboratory.

Morona, R., Mavris, M., Fallarino, A. \& Manning, P. A. (1994). Characterization of the $r f c$ region of Shigella flexneri. J Bacteriol 176, 733-747.

Naide, Y., Nikaido, H., Mäkelä, P. H., Wilkinson, R. G. \& Stocker, B. A. D. (1965). Semirough strains of Salmonella. Proc Natl Acad Sci USA 53, 147-153.

Neidhardt, F. C. \& Umbarger, H. E. (1996). Chemical composition of Escherichia coli. In Escherichia coli and Salmonella: Cellular and Molecular Biology, pp. 13-16. Washington, DC: American Society for Microbiology.

Norrander, J. M., Kempe, T. \& Messing, J. (1983). Construction of improved M13 vectors using oligodeoxynucleotide-directed mutagenesis. Gene 26, 101-106.
Nurminen, M., Hellerqvist, C. E., Valtonen, V. V. \& Mäkelä, P. H. (1971). The smooth lipopolysaccharide character of 1,4,(5),12 and $1,9,12$ transductants formed as hybrids between groups B and D of Salmonella. Eur J Biochem 22, 500-505.

Phillips, T. A., Van Bogelen, R. A. \& Neidhardt, F. C. (1984). Lon gene product of Escherichia coli is a heat-shock protein. $J$ Bacteriol 159, 283-287.

Reeves, P. R., Hobbs, M., Valvano, M. A. \& 8 other authors. (1996). Bacterial polysaccharide synthesis and gene nomenclature. Trends Microbiol 4, 495-503.

Rosenberg, M. \& Court, D. (1979). Regulatory sequences involved in the promotion and termination of RNA transcription. Annu Rev Genet 13, 319-353.

Sambrook, J., Fritsch, E. F. \& Maniatis, T. (1989). Molecular Cloning: a Laboratory Manual, 2nd edn. Cold Spring Harbor, NY : Cold Spring Harbor Laboratory.

Smith, D. B. \& Johnson, K. S. (1988). Single-step purification of polypeptides expressed in Escherichia coli as fusions with glutathione-S-transferase. Gene 67, 31-40.

Xiang, S. H., Hobbs, M. \& Reeves, P. R. (1994). Molecular analysis of the $r f b$ gene cluster of a group D2 Salmonella enterica strain: evidence for its origin from an insertion sequence-mediated recombination event between group E and D1 strains. J Bacteriol 176, 4357-4365.

Yanisch-Perron, C., Vieira, J. \& Messing, J. (1985). Improved M13 phage cloning vectors and host strains: nucleotide sequences of the M13mp18 and pUC19 vectors. Gene 33, 103-119.

Yao, Z. \& Valvano, M. A. (1994). Genetic analysis of the O-specific lipopolysaccharide biosynthesis region $(r f b)$ of Escherichia coli K-12 W3110: identification of genes that confer group 6 specificity to Shigella flexneri serotypes Y and 4- $\alpha$. J Bacteriol 176, 4133-4143.

Zhang, L., Toivanen, P. \& Skurnik, M. (1996). The gene cluster directing antigen biosynthesis in Yersinia enterocolitica serotype $\mathrm{O}: 8$ : identification of the genes for mannose and galactose biosyntheses and the gene for the $\mathrm{O}$-antigen polymerase. Microbiology 142, 277-288.

Received 18 January 1999; revised 24 April 1999; accepted 27 April 1999. 